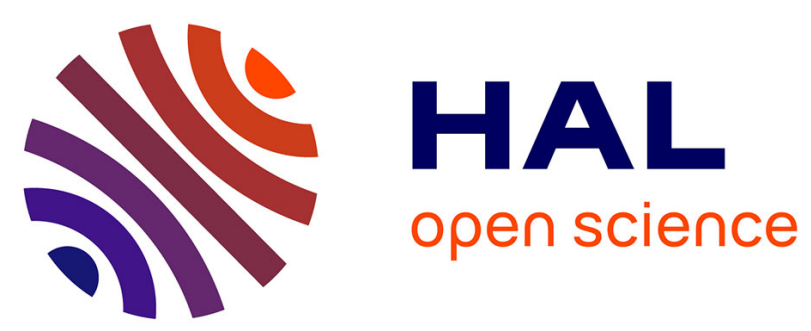

\title{
Financial Markets Dynamics
}

Alain Bretto, Joël Priolon

\section{- To cite this version:}

Alain Bretto, Joël Priolon. Financial Markets Dynamics. 2011. hal-01058611

\section{HAL Id: hal-01058611 \\ https://hal.science/hal-01058611}

Submitted on 27 Aug 2014

HAL is a multi-disciplinary open access archive for the deposit and dissemination of scientific research documents, whether they are published or not. The documents may come from teaching and research institutions in France or abroad, or from public or private research centers.
L'archive ouverte pluridisciplinaire HAL, est destinée au dépôt et à la diffusion de documents scientifiques de niveau recherche, publiés ou non, émanant des établissements d'enseignement et de recherche français ou étrangers, des laboratoires publics ou privés. 


\title{
Financial Markets Dynamics
}

\author{
Alain BRetTo ${ }^{a}$ and Joël PRIOLON ${ }^{b}$ \\ Université de Caen ${ }^{a}$, CNRS-GREYC UMR-6072, Campus II, \\ Bd Maréchal Juin BP 5186, 14032 Caen cedex, France. \\ AgroParisTech; Département des Sciences Économiques, sociales et de gestion ${ }^{b}$ \\ 16, rue Claude Bernard, 75231 Paris Cedex 05, France. \\ alain.bretto@unicaen.fr, joel.priolon@agroparistech.fr
}

\begin{abstract}
The volatility of stock prices is difficult to explain within the confines of rational pricing models. Changes in prices have become permanent at both the individual and the aggregate level. Therefore, when keeping the hypothesis of a rational behavior of agents, we need to give a new explanation to the price settlement of financial assets at any moment of time.

In a model based on an original mathematical framework, we introduce persistent timevarying prices resulting from rational strategic interactions of agents. We demonstrate that in a close to equilibrium market, actual prices give the best approximation of fundamental value; We also explain why, in some circumstances, rational behavior may lead to the development of a bubble or the surge of a financial crisis.
\end{abstract}

\section{Introduction: The two realms of financial economics}

"The special sphere of finance within economics is the study of allocation and deployment of economic resources, both spatially and across time, in an uncertain environment." 1

More specifically it can be stated that most of modern economics of financial markets covers two main fields of research ${ }^{2}$ :

- The first one is dedicated to the modelization of underlying assets markets (stocks, bonds, commodities);

- The second one is mainly concerned with the pricing of derivative assets (futures, options).

From a theoretical point of view, the economics of underlying assets markets tries to understand how equilibrium prices can be settled and, to some extent, how those prices can evoluate over time; We will call $M 1$ the class of economic models aiming at putting light on that category of assets.

Aside of models belonging to $M 1$, another realm of financial economics has been studying the optimal way to estimate the right price of derivative assets. We will call $M 2$ the class of

\footnotetext{
${ }^{1}$ Merton R. C., 1998, Applications of Option-Pricing Theory: Twenty-Five Years Later, The American Economic Review

${ }^{2}$ See one example among so many of them: Bailey R. E., 2007, The Economics of Financial Markets, Cambridge University Press
} 
models dedicated to the valuation of derivative assets. The best known elements of $M 2$ are the Black \& Scholes (B \& S) and the Cox Ross Rubinstein (CRR) models of option pricing. There is absolutely no doubt that both $M 1$ and $M 2$ have known tremendous progress over the last 50 years but some issues remain to be addressed. We stress them shortly hereafter:

- Q1: The valuation of stocks in $M 1$, which is based on present value models, does not appear to be fully compatible with the basic assumptions of B \& S or CRR pricing models. To put it more clearly it can be said that:

- either markets are stable and a price change occurs only when a new information appears (under the assumption of market efficiency) and modifies the expected value of the asset;

- or they evolve continuously ${ }^{3}$ or almost continuously ${ }^{4}$.

Although the two types of assumptions are not mathematically incompatible, from an economic point of view either one considers that markets tend to be stable or that they tend to change permanently. An ever changing stable equilibrium would sound as being a strange concept.

- Q2: If we put attention on the most active markets, such as those of large caps, it may be considered that the development of high frequency trading has deeply transformed the way they work as they tend to become really close to theoretical continuous-time markets, even if actual trades remain discrete. ${ }^{5}$

As a consequence the hypothesis of markets reaching a stable and persistent equilibrium becomes more and more questionable. Therefore, the hypothesis of an ever changing state of the market must be favored and incorporated in the basic assumptions of any market model.

- Q3: Most of the time, markets are driven by forces leading them close to equilibrium prices but, in some special circumstances, markets appear to be dominated by unconventional forces and thus, they step aside from fixed points.

In this paper our intention is to set up a model of financial markets which is able to answer to these yet unanswered questions in a unified framework. To put it shortly, we intend:

- first to explain asset price settlement by strategic behavior of agents, which includes both optimization under constraint and anticipation;

- second to build a causal process relating aggregate outcomes to individual decisions ;

- third to show that most of the time, market dynamics leads to quite ordinary equilibrium paths but, sometimes, may diverge from them thus leading to the development of bubbles or the surge of a crisis.

In section 1 we define the basic lines of our market model; In section 2 we give a set of mathematical definitions which are necessary to understand the mathematical presentation of the model in section 3; Sections 4 and 5 illustrate the core properties of the model.

\footnotetext{
${ }^{3}$ e. $g$. under the assumption of underlying asset prices following a brownian motion

${ }^{4}$ e. $g$. under the assumption of underlying asset prices following a recombinant binomial tree

${ }^{5}$ Just to give an example, on Wednesday July 13 2011, from 9 am to 5 pm, almost 10 thousands transactions have occurred in Amsterdam for Royal Dutch Shell shares.
} 


\section{Market model: Economic assumptions}

The most important building block of our model is the following set of economic assumptions:

- The financial markets are fundamentally driven by what we call a market system (see below our mathematical definition).

- The market system is a dynamic system; At every moment of time it changes because of permanent revisions of agents' behavior.

- Most of the time the market system is itself determined by ordinary economic fundamental forces (what we will call objective factors). Thus, most of the time, the market system follows an equilibrium path. For instance, asset prices tend to be close to the fundamental value of the asset.

- In some situations such as a crisis, the market system may be driven by some other forces (what we call subjective factors).

- Because of permanently revised anticipations of interacting agents, the market system is never stuck in a non evolving equilibrium. Thus, even in situations of stability, the market never reaches an inert equilibrium. This is why, for example, asset prices are permanently changing although the market appears to be globally stable.

We now turn to the core economic definition of our model which is the concept of market system. A mathematical definition of this concept will be given in section 3 .

\section{$1.1 \quad$ Market system}

A market system is a set of functions which determine the dynamics of the financial market. These functions depend on parameters which may be objective or subjective (see definitions below). The core of the model are the agents who make observations, who calculate, anticipate and who act as buyers or sellers of financial assets. What makes the difference between our model and usual microeconomic models relates at least to the two following points.

\subsubsection{Individual behaviour}

Although agents are supposed to be percfectly rational, in some circumstances they cannot rely on ordinary economic calculus to make their decisions. Therefore they use other criteria who do not belong to conventional optimisation tools. In other words we consider that economic decision is not only determined by a kind of mechanical behaviour but also depends of different other parameters.

As a main consequence, in a stable environment with negligible or low uncertainty, the market almost works like any equilibrium market of general microeconomics; Thus general results of microeconomics of financial markets remain valid. But, in a more turbulent environment, we are able to give an explanation of the market dynamics out of ordinary equilibrium.

\subsubsection{Aggregate behaviour}

One of the most difficult tasks of social sciences is to relate the global outcomes to the

individual behaviours. A very widespread technique consists in assuming that a representative 
agent can summarize the behaviour of a large number of individual agents.

Although this technique appears to be convenient, it remains unsatisfactory as it leaves aside the modelisation of aggregate outcome even if the properties of individual agents are precisely known. In our model we are able to tell at any moment what the outcome of multiple agents interactions will be.

\subsection{Market dynamics and market outcomes}

As a result of the dynamics of the underlying market system and at any moment of time we can make some observations. We will call quantifiable properties the set of measures that we can make; These quantifiable properties belong to the following (open) list: prices, traded quantities, elasticities, bid ask spreads, number of orders, minimum and maximum prices, and so on.

\subsubsection{Equilibrium paths}

On an equilibrium path, the observed prices of actual trades are close to fundamental values which are determined by present value models; Thus actual prices gravitate around the true price of the assets.

One theoretical gain of our model over more standard microeconomic one is the fact that we are able to explain small variations around equilibrium prices, even if we keep the rational expectation hypothesis. Small variations can be interpreted as the result of the unavoidable persistence of subjective factors, even if those factors have normally a very small influence on the outcome of the market.

\subsubsection{Out of equilibrium paths}

When uncertainty becomes too high the economic agents become unable to make their decisions on the basis of a simple economic calculus. Therefore prices are no more determined by the fundamental value of the asset; Different kinds of factors overwhelm objective parameters of the market.

One theoretical gain of our model is our ability to give a rational explanation to the use of subjective factors. Thus we can keep the hypothesis that individuals act rationally, even in situations where the market diverges from equilibrium.

\section{Mathematic preliminaries and definitions}

This section is exclusively dedicated to a set of mathematical preliminaries and definitions which have no direct economic meaning but which are necessary to develop some demonstrations in the section 3 .

Our main task is to give a mathematical definition of what most of the time precisely means because we need to define a measure which makes it possible to compare situations in which the market is driven by objective factors to situations where subjective factors become determinant. 


\subsection{Definition of a measure $\mu$}

\section{In the sequel map is equivalent to function.}

In our model, we need to use a measure in order to compare different sets or subsets. The object of this subsection is to give a definition of the measure $\mu$ that we will use.

Let $\mathcal{S}$ be a non-empty subset of the power set of $X$ closed under the union of pairs of sets and under complementary set with $X$ belonging to $\mathcal{S}$. The couple $(X ; \mathcal{S})$ is called a field of sets or an algebra of sets. In other words $(X ; \mathcal{S})$ is an algebra of sets if :

1): $X \in \mathcal{S}$;

2): If $A \in \mathcal{S}$ then $\bar{A} \in \mathcal{S}$, where $\bar{A}=X \backslash A$;

3): If $A, B \in \mathcal{S}$ then $A \cup B \in \mathcal{S}$.

Let $X$ be a set and $\mathcal{F} \subseteq 2^{X} ; \mathcal{F}$ is a $\sigma$-algebra if it verifies:

1): $X \in \mathcal{F}$;

2): If $A \in \mathcal{F}$ then $\bar{A} \in \mathcal{F}$;

3): If $\left(A_{i}\right)_{i \in I}$ is a countable family of elements of $\mathcal{F}$ then $\bigcup_{i} A_{i} \in \mathcal{F}$.

The set $(X ; \mathcal{F})$ is called measurable space. It is rather easy to prove the following:

Lemma 1. Let $X$ be a set and let $(\mathcal{S})_{i}$ be a countable family of algebras of sets, (resp. a countable family of $\sigma$-algebras) on $X$, then $\cap_{i} \mathcal{S}_{i}$ is an algebra of sets (resp. is a $\sigma$-algebra).

Let $X$ be a set and $\mathcal{F} \subseteq 2^{X}$; the algebra of sets generated, (resp. the $\sigma$-algebra generated) by $\mathcal{F}$ is the intersection of all algebras of sets, (resp. of all $\sigma$-algebras) containing $\mathcal{F}$.

Let $\mathcal{F}$ be an algebra of sets (a $\sigma$-algebra) over a set $X$. A function $\mu$ from $\mathcal{F}$ to $[0 ;+\infty]$ is called a measure if it satisfies the following properties:

a): Null empty set: $\mu(\varnothing)=0$;

b): Countable additivity (or $\sigma$-additivity): For all countable collections $\left\{A_{i}\right\}_{i \in I}$ of pairwise disjoint sets in $\mathcal{F}$, we have $\mu\left(\bigcup_{i \in I} A_{i}\right)=\sum_{i \in I} \mu\left(A_{i}\right)$.

A measurable space $(X ; \mathcal{F})$ with a measure $\mu$ is called a measure space; We denote it by: $(X ; \mathcal{F} ; \mu)$.

Let $(X ; \mathcal{F} ; \mu)$ be a measure space, a subset $Y$ of $X$ is negligible if there is a $A \in \mathcal{F}$ such that $Y \subseteq A$ and $\mu(A)=0$.

For $A \in X$ If $\mu(A)<+\infty$ we say that $A$ has a finite measure.

Let $(X ; \mathcal{F} ; \mu)$ be a measure space, then the measure $\mu$ is called finite if $\mu(X)$ is a finite real number; The measure $\mu$ is called $\sigma$-finite if $X$ can be decomposed into a countable union of measurable sets of finite measure. 


\section{$2.2 \quad$ Properties true almost everywhere}

Let $(X ; \mathcal{F} ; \mu)$ be a measure space. For all $x \in X$, let $P(x)$ be a property of $(X ; \mathcal{F} ; \mu)$; this property is true almost everywhere if the set $\{x \in X\}$ where this property is not true is negligible.

Let

$$
\begin{array}{rll}
f, g: I \subseteq[0 ; 1] & \longrightarrow V \\
t & \mapsto o(t)
\end{array}
$$

be two functions.

The function $f$ is neglible before the function $g$ at $a \in I$ if there is a neighborhood $W$ of $a$ and a function

$$
\begin{aligned}
\epsilon: \quad W & \longrightarrow V \\
t & \mapsto \epsilon(t)
\end{aligned}
$$

such that for all $t \in W, f(t)=\epsilon(t) . g(t), \lim _{t \longrightarrow a} \epsilon(t)=0$ and $\lim _{t \longrightarrow a} g(t) \neq 0$. We will denote this by $f=o(g)$.

We say that the function $f$ is neglible before the function $g$ almost everywhere on $I \subseteq[0 ; 1]$ if there is a sequence $\left(a_{k}\right), a_{k} \in I$ and a sequence of neighborhood $\left(W_{a_{k}}\right)$ with $I \subseteq \bigcup_{a_{k}} W_{a_{k}}$ where $f$ is negligible for all $a_{k} \in I$ except on a set $Y \subseteq I$ where there exists a measure $\mu$ such that $Y$ is negligible for $\mu$. We denote this by $f=o_{\text {a.e. }}(g)$.

A function $f$ is equivalent to a function $g$, denoted by $f \sim g$ at $a \in I$ if there is a neighborhood $W$ of $a$ and a function $\epsilon$ defined as above such that for all $t \in W, f(t)=(1+\epsilon(t)) \cdot g(t)$, $\lim _{t \longrightarrow a} \epsilon(t)=0$. This relation is an equivalent relation. It is easy to see that $(f \sim g) \Longleftrightarrow$ $(f-g=o(g)) \Longleftrightarrow(g-f=o(f))$. In the same way as above we say that a function $f$ is equivalent to a function $g$ almost everywhere on $I \subseteq[0 ; 1]$; we will denote this relation as follows: $f \sim$ a.e. $g$.

Naturally we can extend these definitions to vector functions defined in a same basis; It is enough to check the conditions for all coordinates $\left(f_{1}(t), f_{2}(t), \ldots f_{k}(t)\right)$, where $k$ is the dimension of the vector space.

More generally let $H$ be a space of vectors with 2 basis $\left(e_{1}, e_{2}, \ldots e_{n}\right)$ and $\left(a_{1}, a_{2}, \ldots a_{n}\right)$; Two vectors depending of a variable $t$, (for example the time) are equivalent at $t: v(t) \sim$ $w(t) \Longleftrightarrow \sum_{j=1}^{n} \beta_{j} e_{j} \sim \sum_{j=1}^{n} \alpha_{j} a_{j}$ if and only if $\beta_{j} \sim \alpha_{j}$ and $e_{j} \sim a_{j}$, (we drop the variable $t$ to avoid burdening the notations).

\section{$2.3 \quad$ Piecewise effective functions}

An algorithm is a finite sequence of finite instructions.

A real number is called effectif, if there exists an algorithm which can produce its decimal expansion. By extension a complex number is effectif if there exists an algorithm which can produce the decimal expansion of both the real part and the imaginary part. We can also extend these defintions to a vector function. 
Let $f: X \longrightarrow Y$ be a function such that $X$ is a set of effective elements (real, complex, vectors, ...). This function $f$ is effective if $\forall z \in X, f(z)$ is effective i. e. the ordered pair $(z ; f(z))$ is an ordered pair of effective numbers for all $z$ of the domain of $f$. Notice that we can combine this definition with the definition of almost everywhere to get effective function almost everywhere.

Addition, multiplication and composition of effective functions are effective functions. Morevover addition, multiplication, ... of effective reals, complexes, vectors are effective reals, complexes, vectors. So the set of real numbers, (complex numbers) forms a field denoted by $\mathbb{R}_{e f},\left(\mathbb{C}_{e f}\right)$. The zero function will be denoted by $\mathbb{O}$ : for all $x$ of the domain of $\mathbb{O}, \mathbb{O}(x)=0$.

A function $f$ is piecewise effective on $[a, b]$ if there exists a subdivision $\left(a_{i}\right)$ of $[a, b]$ such that the restriction of $f$ to each $] a_{i}, a_{i+1}[$ can be prolonged by a function effective on the corresponding closed set $\left[a_{i}, a_{i+1}\right]$

\section{Single Market Model: Axiomatic and First Results}

From an economic point of view, one of our basic assumptions is that the market is driven by fundamental forces. We call market system the set of factors who determine the dynamics of the market.

In the case of financial markets it appears that the model must be built in three steps:

- We consider first a single market system which will be defined as the market for a singular bond, a singular share or any other financial security listed on a market;

- Second, a financial market compartiment will be defined as the union of several single markets who share something in common;

- Ultimately, The financial market may be considered as the union of all single markets.

In the following part of the paper we concentrate exclusively on the definition of a single market system, although we give some short views on the possibilities of generalizing the model.

A single market system-SMS- is an element of the global financial markets that we modelize independently of the other elements. For instance, we will limit our analysis to the market of one share traded on an Exchange. Thus me make the assumption that, in a first approach, this single market may be studied independently of the market of other shares traded on the same Exchange or on another Exchange.

This means that every single market is homogeneous. We assume that every market system is a fully determined system, although we are not able to access to a perfect knowledge about it.

The fundamental single market system is defined as follows: At any time $t$ and for any single market system there exists a set of intrinsic characteristics which determine this single market system. From this fundamental market system we can deduce for instance the fundamental value of one share.

We will see more details about these values later. We now turn economic assumptions into mathematical axioms.

Axiom 1. The fundamental single market system exists. 
It means that some underlying fundamental forces determine the market system dynamics; It does not mean however that the market spontaneously reaches a stable equilibrium or a stable state.

Axiom 2. A single market system is completely defined at the time $t$ by a (state) unitary vector $m s(t)$ or $m s$ belonging to a Hilbert space on $\mathbb{C}$. This space will be denoted by $\mathcal{H}$.

We need to make calculations: analyticals, algebraics, vectors, .... So we need an underlying space which allows us to make these calculations. An Hilbert space appears to be a good candidate for this purpose.

Axiom 3. To each quantifiable property of a market system (price, quantities,....) $M$ corresponds a linear operator $\widetilde{M}$ which acts on $\mathcal{H}$; The only possible outcomes of measurement (evaluation, reading) of the quantifiable property $M$ are eigenvalues of $\widetilde{M}$.

Remark 1. The system of vectors ms $(t)$, with $t \in[0 ; 1]$ (ms for short) represents a function called single market system function.

Whithout losing generality we can suppose that any measurement of a quantifiable property is a positive real number, consequently the eigenvalues of $\widetilde{M}$ exist and must be real and positive. Hence $\widetilde{M}$ is a definite positive hermitian operator.

Consequently, the measurement (evaluation or reading) of the quantifiable property $M$ is internal to the market theory.

Axiom 3 implies that there is a fundamental determination of the evolution of the market. However, the observation of the system reveals just a part of the dynamics of the market system.

Let $M$ be a quantifiable property of a market system and $\widetilde{M}$ be the linear operator associated with it. The Eigenvectors of $\widetilde{M}:\left(e_{i}\right)_{i \in\{1,2, \ldots n\}}$ are an orthonormal basis of $\mathcal{H}$.

Thus, $m s=\sum_{i} \beta_{i} e_{i}$.

Axiom 4. Let a market system be described as follows : $m s=\sum_{i} \beta_{i} e_{i}$.

Then, the probability that the result of the measurement of the size $M$ at the time $t$ be $\lambda_{i}$ is $\sum_{i=1}^{k_{\lambda_{i}}}\left|\beta_{i}\right|^{2}$, where $k_{\lambda_{i}}$ is the number of eigenvectors, (linearly independant) associated with the eigenvalue $\lambda_{i}$. In other words, $\operatorname{Pr}$ (Measurement of $\left.M=\lambda_{i}\right)=\sum_{i=1}^{k_{\lambda_{i}}}\left|\beta_{i}\right|^{2}$.

A parameter of the single market system denoted by $p$ is a function capable of changing a single market system.

An objective parameter (resp. subjective parameter) is a parameter of a SMS independent of any human activity (resp. dependent of the human activities).

We will represent these parameters by the following functions:

$$
\begin{array}{lll}
o: \quad I \subseteq[0 ; 1] & \longrightarrow & V \\
t & \mapsto
\end{array}
$$


the function $o$ is an effective function and it belongs to the piecewise class $C^{1}$

$$
\begin{array}{rll}
s: \quad I \subseteq[0 ; 1] & \longrightarrow & V \\
t & \mapsto s(t)
\end{array}
$$

the function $s$ is not an effective function and it is piecewise continuous.

The set $V$ is a linear space with a finite dimension, and $I$ is the union of disjoint segments.

Theorem 1. The set of parameters of the single market system, objective parameters and subjective parameters equipped with zero function form linear spaces on $\mathbb{C}_{\text {ef }}$ denoted by $\mathcal{P}(I ; V)$, $\mathcal{O}(I ; V)$ and $\mathcal{S}(I ; V)$. Moreover :

- $\mathcal{P}(I ; V)=\mathcal{O}(I ; V) \oplus \mathcal{S}(I ; V)$.

Proof. The sum and the product by an effective complex number of objective parameters (effective functions) is an objective parameter (effective function) and the sum and the product by a complex number of subjective parameters (not effective functions) is a subjective parameter (not effective function) ; The sum and the product by an effective complex number of functions in piecewise class $C^{1}$ belong to piecewise class $C^{1}$. So these sets form linear spaces, idem for piecewise continuous function.

Moreover we have: $\mathcal{O}(I ; V) ; \mathcal{S}(I ; V) \leqslant \mathcal{P}(I ; V)$. Since an objective parameter is not subjective and subjective parameter is not objective we have: $\mathcal{O}(I ; V) \cap \mathcal{S}(I ; V)=\{\mathbb{O}\}$.

Let $p \in \mathcal{P}(I ; V), p$ has an objective component (effective component) and a subjective component (either non effective component or $\mathbb{O}$ ), consequently $p=o+s$ where $o \in \mathcal{O}(I ; V)$ and $s \in \mathcal{S}(I ; V)$.

Let $\left(t_{k}\right)_{k \in\{0,1,2, \ldots\}=K \subseteq \mathbb{N}}$ be a strictly monotone countable sequence of elements of $[0 ; 1]$ such that $t_{0}=0$. Let $I=\bigcup_{l \in L \subseteq K}\left[t_{2 . l} ; t_{2 . l+1}\right]$. Define on $I$ an algebra of sets $\mathcal{S}$ in the following way:

- $\varnothing \in \mathcal{S}$

- For all $l \in L$, all $t_{1} ; t_{2} \in\left[t_{2 . l} ; t_{2 . l+1}\right]$ then $\left[t_{1} ; t_{2}\right] \in \mathcal{S}$;

- if $\left[t_{1} ; t_{2}\right] \in \mathcal{S}$ then $\overline{\left[t_{1} ; t_{2}\right]} \in \mathcal{S}$;

- if $I_{1}, I_{2} \in \mathcal{S}$ then $I_{1} \cup I_{2} \in \mathcal{S}$.

Denote by $\mathcal{F}=\sigma(\mathcal{S})$ the $\sigma$-algebra generated by $\mathcal{S}$.

Let

$$
\begin{aligned}
F: \quad I \subseteq & \longrightarrow \mathbb{R} \\
t & \mapsto F(t)
\end{aligned}
$$

be a monotone map, (I. e. $t_{1} \leqslant t_{2} \Longrightarrow F\left(t_{1}\right) \leqslant F\left(t_{2}\right)$ ). Define a map $\mu: \mathcal{S} \longrightarrow[0 ;+\infty]$ in the following way:

a): For $\left(I_{j}\right)_{j \in J}=\left(\left[t_{j} ; t_{j+1}\right]\right)_{j \in J}$ with $\left[t_{j} ; t_{j+1}\right] \in \mathcal{S}$ such that for all $j_{1}, j_{2} \in J, I_{j_{1}} \cap I_{j_{2}}=\varnothing$ then

$$
\mu\left(\bigcup_{j \in J}\left[t_{j} ; t_{j+1}\right]\right)=\sum_{j \in J} F\left(t_{j+1}\right)-F\left(t_{j}\right) ;
$$


b):

$$
\mu(\varnothing)=0
$$

Lemma 2. The map $\mu$ defines a positive $\sigma$-finite measure on $(I ; \mathcal{S})$.

Proof. We have to show that $\mu\left(\overline{\left[t_{i} ; t_{i+1}\right]}\right)$ exists.

Let $t_{1}, t_{2} \in\left[t_{2 . l^{\prime}} ; t_{2 . l^{\prime}+1}\right]$. Hence $\left[t_{2 . l^{\prime}} ; t_{2 . l^{\prime}+1}\right]=\left[t_{2 . l^{\prime}} ; t_{1}\right] \cup\left[t_{1} ; t_{2}\right] \cup\left[t_{2} ; t_{2 . l^{\prime}+1}\right]$. So $\overline{\left[t_{1} ; t_{2}\right]}=$ $\left[t_{2 . l^{\prime}} ; t_{1}\right] \cup\left[t_{2} ; t_{2 . l^{\prime}+1}\right] \cup\left(\bigcup_{l \in L, l \neq l^{\prime}}\left[t_{2 . l} ; t_{2 . l+1}\right] . \quad\right.$ Consequently: $\mu\left(\overline{\left[t_{i} ; t_{i+1}\right]}\right)=\mu\left(\left[t_{2 . l^{\prime}} ; t_{1}\right]\right)+$ $\mu\left(\left[t_{2} ; t_{2 . l^{\prime}+1}\right]\right)+\sum_{l \in L, l \neq l^{\prime}} \mu\left(\left[t_{2 . l} ; t_{2 . l+1}\right]\right)$. Remark that if $\overline{\left[t_{1} ; t_{2}\right]}, \overline{\left[t_{3} ; t_{4}\right]} \in \mathcal{S}$ with $\overline{\left[t_{1} ; t_{2}\right]} \cap$ $\overline{\left[t_{3} ; t_{4}\right]}=\varnothing$ then $\overline{\left[t_{1} ; t_{2}\right]}=\left[t_{3} ; t_{4}\right]$ and $\overline{\left[t_{3} ; t_{4}\right]}=\left[t_{1} ; t_{2}\right]$, so $\left[t_{1} ; t_{2}\right] \cup\left[t_{3} ; t_{4}\right]=I$ and $\left[t_{1} ; t_{2}\right] \cap$ $\left[t_{3} ; t_{4}\right]=\varnothing$. Consequently: $\mu\left(\overline{\left[t_{1} ; t_{2}\right]} \cup \overline{\left[t_{3} ; t_{4}\right]}\right)=\mu\left(\overline{\left[t_{1} ; t_{2}\right]}\right)+\mu\left(\overline{\left[t_{3} ; t_{4}\right]}\right)$.

Moreover $F$ being monotone we have: $F\left(t_{j+1}\right)-F\left(t_{j}\right) \geqslant 0$. This measure is $\sigma$-finite since $I=\bigcup_{l \in L \subseteq K}\left[t_{2 . l} ; t_{2 . l+1}\right]$ which is a countable union and $\mu\left(\left[t_{2 . l} ; t_{2 . l+1}\right]\right)<+\infty$. for all $l \in L$

Proposition 1. Let $\mathcal{S}$ be an algebra of sets defined on $I$ and $\mu$ be the measure on $\mathcal{S}$ defined above. There is a unique measure $\mu^{\prime}$ on $\sigma(\mathcal{S})=\mathcal{F}$ which is a unique extension of $\mu$ on $\mathcal{S}$, ( $i$. e. $\left.\mu_{\mid \mathcal{S}}^{\prime}=\mu\right)$.

Proof. From lemma 2, the measure $\mu$ is positive; moreover it is $\sigma$-finite, by applying HAHN extension theorem, ([13]) we have the result.

In the sequel without losing generality we denote $\mu^{\prime}$ by $\mu$.

Axiom 5. For all parameters of the single market system $p_{i}=o_{i}+s_{i}$, we have $s_{i}=\mathrm{o}_{a . e .}\left(o_{i}\right)$ for the measure defined above.

We consider that, most of the time, the market system is mainly or even fully determined by the objective parameters ; But, in some circumstances such as financial crisis, subjective parameters may take precedence over objective ones.

\subsection{Market system representation}

Let $\left(p_{i}\right)_{i \in\{1,2, \ldots k\}}$, be a family of parameters and $\left(o_{i}\right)_{i \in\{1,2, \ldots k\}}$ (resp. $\left.\left(s_{i}\right)_{i \in\{1,2, \ldots k\}}\right)$ be a family of objective parameters (resp. subjective parameters). We can redefine a market system as a function $f$ in the following way:

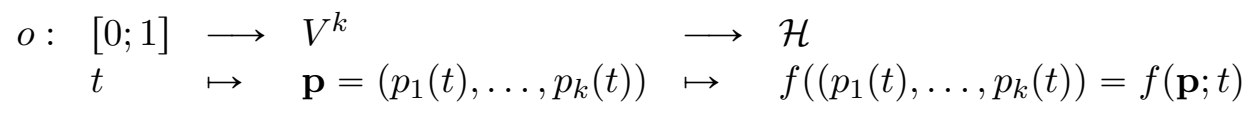

The value of $f\left(\left(o_{1}(t), \ldots, o_{k}(t)\right)=f(\mathbf{o} ; t)\right.$ is the objective fundamental market system and the value $f\left(s_{1}(t), \ldots, s_{l}(t)\right)=f(\mathbf{s} ; t)$ is the subjective fundamental market system.

An important remark: When speaking of a subjective fundamental market system, we do not refer to any form of irrationality. It only means that in the case of an objective value, an optimisation calculus can be operated; In the case of a subjective fundamental market system the imperfectness of information makes it impossible to execute such a calculus; Then the agents must rely on other criteria to make their decisions.

Remark 2. To each time $t$ fixed, when there is no ambiguity we can write $m s=f(\mathbf{p})$, (idem for $m s_{o}=f(\mathbf{o})$ and $\left.m s_{s}=f(\mathbf{s})\right)$, hence we can see a single market system as a vector or a function. Since these vectors are unitary, a single market system is described on a sphere 
called market sphere.

Let $M$ be a quantifiable property at the time $t \in I$. We can associate 3 linear operators: $\widetilde{M}_{m s}$, $\widetilde{M}_{m s_{o}}$ and $\widetilde{M}_{m s_{s}}$.

Proposition 2. By using above notations we have: $f(\mathbf{p} ; t) \sim_{a . e .} f(\mathbf{o} ; t)$.

Proof. Let $a \in I$ such that $s_{i}=o\left(o_{i}\right)$ in a neighborhood of $a$ for all $i \in\{1,2, \ldots k\}$ and $f_{p}(t)=f\left(p_{1}(t), \ldots, p_{k}(t)\right)$. We have:

$\lim _{t \rightarrow a} \frac{f_{p}(t)}{\left.f_{o}(t)\right)}=\lim _{t \rightarrow a} \frac{f\left(p_{1}(t), \ldots, p_{k}(t)\right)}{f\left(o_{1}(t), \ldots, o_{k}(t)\right)}=\lim _{t \rightarrow a} \frac{f\left(o_{1}(t)+s_{1}(t), \ldots, o_{k}(t)+s_{k}(t)\right)}{f\left(o_{1}(t), \ldots, o_{k}(t)\right)}=$ $\lim _{t \rightarrow a} \frac{f\left(o_{1}(t)+\epsilon_{1}(t) o_{1}(t), \ldots, o_{k}(t)+\epsilon_{1}(t) o_{k}(t)\right)}{f\left(o_{1}(t), \ldots, o_{k}(t)\right)}=\frac{f\left(o_{1}(a), \ldots, o_{k}(a)\right)}{f\left(\left(o_{1}(a), \ldots, o_{k}(a)\right)\right.}=1 \Longleftrightarrow f(p) \sim f(o)$.

Let $b \in I$ such that for all neighborhood $W$ of $b, f(\mathbf{p} ; t) \nsim f(\mathbf{o} ; t)$ for $t \in W$. It is equivalent to $\lim _{t \rightarrow b} \frac{f\left(p_{1}(t), \ldots, p_{k}(t)\right)}{f\left(o_{1}(t), \ldots, o_{k}(t)\right)} \neq 1$. Hence there is $i \in\{1,2,3, \ldots k\}$ such that there is no $\epsilon_{i}$, with $s_{i}(t)=\epsilon_{i}(t) o_{i}(t)$ and $\lim _{t \rightarrow b} \epsilon_{i}(t)=0$. Consequently, from axiom 5 we have: $b \in Y \subseteq A \subseteq I$ such that $\mu(A)=0$.

So we can conclude that: $f(\mathbf{p} ; t) \sim_{\text {a.e. }} f(\mathbf{o} ; t)$.

Theorem 2 and Corollary 1 will give some important illustrations of Proposition 2.

Axiom 6. If the reading of a quantifiable $M$ gives the eignenvalue $\lambda_{i}$ of $\widetilde{M}$ then the state of the single market system instantly after the observation of $M$ is

$$
f_{\lambda_{i}}(\mathbf{p})=\frac{1}{\sum_{j=1}^{k_{\lambda_{i}}}\left|\beta_{j}\right|^{2}} \sum_{j=1}^{k_{\lambda_{i}}} \beta_{j} e_{j}
$$

Idem for subjective, (resp. objective) single market system.

Remark 3. This axiom says that if the reading of a quantifiable is the eigenvalue $\lambda_{i}$ then instantly the market system is in the vector space spanned the eigenvectors associated to $\lambda_{i}$. It is a projection on this vector space.

This axiom means that, on the one hand, the economic agents behavior determines the market outcomes and that, on the other hand, the state of the market system has an influence on the agents' decisions.

In standard microeconomic models, demand is a monotonous decreasing function of prices (without asymptote) and offer is a monotonous growing function of prices (without asymptote). The equilibrium price is the intersection between the two functions. ${ }^{6}$ Thus the price is mechanically determined, it is not the result of a strategic behavior.

In our model, the settlement of prices in time $t$ depends of anticipations in time $t-1$. More precisely the $N$ agents $e_{i}(i=1 \ldots n)$ make anticipations denoted $\lambda_{i}$ which are the eigenvalues of the linear operator associated to a quantifiable (for instance the asset price). In time $t$ one of the anticipations becomes true; Then the market system curve adjusts almost instantly to this outcome. From a mathematical point of view, this value is a projection on the space of $\lambda_{i}$.

Then, once the new state of the market in $t$ is known, the new set of information makes it possible for agents to develop new anticipations for time $t+1$.

\footnotetext{
${ }^{6}$ Moreover, the market is supposed to be cleared only when the equilibrium price has been settled
} 
Remark: At the limit, in a situation of absolutely perfect information and under the assumption of perfect rationality of agents, all the $\lambda_{i}$ may be identical.

This axiom is not artificial: for example suppose you're a bookmaker, you know that a great champion runs in the next race, your prognostic is that it will win the race with a probability of $90 \%$. A few minutes before departure you learn that this great champion just made a slight sprain, then your probability that this champion wins the race passes instantly from $90 \%$ to nearly $0 \%$.

Theorem 2. Let $J=\left[t_{1} ; t_{2}\right] \subseteq I$ such that $\mu(] t_{1} ; t_{2}[) \neq 0$. Let $M$ be a quantifiable property at the time $t \in] t_{1} ; t_{2}\left[\right.$, let $\widetilde{M}_{m s}, \widetilde{M}_{m o}$ be the linear operators associate with $M$. If that the eigenvalues of $\widetilde{M}_{m s}$ are $\lambda_{1}>\lambda_{2} \geqslant \lambda_{3} \geqslant \ldots \lambda_{n-1}>\lambda_{n}$ and the eigenvalues of $\widetilde{M}_{m o}$ are $\mu_{1}>\mu_{2} \geqslant \mu_{3} \geqslant \ldots \mu_{n-1}>\mu_{n}$ then there is a "small" $\epsilon$ such that $\left|\lambda_{i}-\mu_{i}\right| \leqslant \epsilon$ for all $i \in\{1,2,3, \ldots, n\}$.

Proof. Let $\left(e_{i}\right)_{i \in\{1,2, \ldots n\}}$ and $\left(a_{i}\right)_{i \in\{1,2, \ldots . n\}}$ be the basis of orthonormal eigenvectors associated to $\left(\lambda_{j i}\right)_{i \in\{1,2, \ldots n\}}$ and $\left(\mu_{j i}\right)_{i \in\{1,2, \ldots n\}}$ respectively.

By applying axiom 5 and proposition 2 we have $f\left(\mathbf{p} ; t^{\prime}\right) \sim f\left(\mathbf{o} ; t^{\prime}\right)$ for all $t^{\prime} \in J$, since $\mu\left(\left[t_{1} ; t_{2}\right]\right) \neq 0$. For $\left.t \in\right] t_{1} ; t_{2}\left[\right.$ we have: $f(\mathbf{p}) \sim f(\mathbf{o}) \Longleftrightarrow \sum_{j=1}^{n} \beta_{j} e_{j} \sim \sum_{j=1}^{n} \alpha_{j} a_{j} \Longleftrightarrow$ $m s \sim m o \sim \sum_{i=1}^{n} \beta_{i} e_{i}$.

If $\widetilde{M}_{m s}=\widetilde{M}_{m o}$, it is over.

Assume that $\widetilde{M}_{m s} \neq \widetilde{M}_{m o}$.

$m s(k)=\left(\widetilde{M}_{m s}\right)^{k} m s=\sum_{i=1}^{n} \lambda_{i}^{k} \beta_{i} e_{i}=\lambda_{i}^{k}\left[\beta_{1} e_{1}+\sum_{i=2}^{n} \beta_{i}\left[\frac{\lambda_{i}}{\lambda_{1}}\right]^{k} e_{i}\right]=\lambda_{i}^{k}\left[\beta_{1} e_{1}+\mathcal{O}\left(\left[\frac{\lambda_{i}}{\lambda_{1}}\right]^{k}\right)\right]$.

$m o(k)=\left(\widetilde{M}_{m o}\right)^{k} m o=\sum_{i=1}^{n} \mu_{i}^{k} \beta_{i} e_{i}=\mu_{i}^{k}\left[\beta_{1} e_{1}+\sum_{i=2}^{n} \beta_{i}\left[\frac{\mu_{i}}{\mu_{1}}\right]^{k} e_{i}\right]=\mu_{i}^{k}\left[\beta_{1} e_{1}+\mathcal{O}\left(\left[\frac{\mu_{i}}{\mu_{1}}\right]^{k}\right)\right]$.

Let's $\widetilde{m s(k)}=\frac{\left(\widetilde{M}_{m s}\right)^{k} m s}{\left\|\left(\widetilde{M}_{m s}\right)^{k} m s\right\|}$ and $\widetilde{m o(k)}=\frac{\left(\widetilde{M}_{m o}\right)^{k} m o}{\left\|\left(\widetilde{M}_{m o}\right)^{k} m o\right\|}$.

We have: $\widetilde{m s(k)}=\frac{\lambda_{i}^{k}\left[\beta_{1} e_{1}+\mathcal{O}\left(\left[\frac{\lambda_{i}}{\lambda_{1}}\right]^{k}\right)\right]}{\left\|\lambda_{i}^{k}\left[\beta_{1} e_{1}+\mathcal{O}\left(\left[\frac{\lambda_{i}}{\lambda_{1}}\right]^{k}\right)\right]\right\|} \simeq \frac{\beta_{1} e_{1}}{\left|\beta_{1}\right|}$ when $k \longrightarrow+\infty$; we have also : $\widetilde{m o(k)} \simeq \frac{\beta_{1} e_{1}}{\left|\beta_{1}\right|}$

when $k \longrightarrow+\infty$. Consequently when $k \longrightarrow+\infty$ we have: $\widetilde{m s(k)} \simeq \widetilde{m o(k)}$, hence $\left.\widetilde{M}_{m s}\right)^{k} m \simeq$ $\frac{\left\|\left(\widetilde{M}_{m s}\right)^{k} m s\right\| \cdot\left(\widetilde{M}_{m o}\right)^{k} m o}{\left\|\left(\widetilde{M}_{m o}\right)^{k} m o\right\|}$; it is equivalent, when $k \longrightarrow+\infty$ to $\lambda_{1}^{k} \beta_{1} e_{1} \simeq t \cdot \mu_{1}^{k} \beta_{1} e_{1}$ So $\lambda_{1}^{k} \simeq t$. $\mu_{1}^{k}$. So since $\lambda_{1}, \mu_{1}, t>1$ and $t^{\frac{1}{k}} \longrightarrow 1$ when $k \longrightarrow+\infty$ we have:

$$
\lambda_{1} \simeq \mu_{1} \text {. }
$$

From remark 1: $\widetilde{M}_{m s}, \widetilde{M}_{m o}$ are invertible and when $t^{\prime} \longrightarrow t: e_{i}=a_{i}$ for all $i \in\{1,2, \ldots, n\}$; moreover if $e_{i}$ are eigenvectors of $\widetilde{M}_{m s}$ and $\widetilde{M}_{m o}$ then $e_{i}$ are also eigenvectors of $\widetilde{M}_{m s}^{-1}$ and $\widetilde{M}_{m o}^{-1}$.

Choose $\alpha \in \mathbb{R}$ such that $\alpha<\lambda_{n}, \alpha<\mu_{n}, 0<\lambda_{n}-\alpha<\lambda_{i}-\alpha$ and $0<\mu_{n}-\alpha<\mu_{i}-\alpha$ for all $i \in\{1,2, \ldots, n-1\}$.

Let's $\widetilde{M}_{m s}-\alpha . I, \widetilde{M}_{m o}-\alpha . I$; these two matrices have as eigenvalues $\lambda_{i}-\alpha, \mu_{i}-\alpha$ and the eigenvectors associates with them are $e_{i}, i \in\{1,2, \ldots, n\}$. These two matrices are invertible. From this: $\left(\lambda_{i}-\alpha\right)^{-1},\left(\mu_{i}-\alpha\right)^{-1}, i \in\{1,2, \ldots, n\}$ are eigenvalues of $\widetilde{M}_{m s}^{-1}$ and $\widetilde{M}_{m o}^{-1}$ associated to the eigenvectors $e_{i}$. By applying the same argumentation as above we have: $\left(\lambda_{n}-\alpha\right)^{-1} \simeq$ $\left(\mu_{n}-\alpha\right)^{-1}$. Consequently:

$$
\lambda_{n} \simeq \mu_{n} .
$$

Let us $\delta(\widetilde{M})=\widetilde{M}_{m s}-\widetilde{M}_{m o}$; since these two matrices are hermitian and $\widetilde{M}_{m s} \neq \widetilde{M}_{m o}$ then $\delta(\widetilde{M})$ is hermitian; hence $\delta(\widetilde{M})$ is diagonalizable and its eigenvalues are real. 
From inequality of WEYL [4] we have:

$$
\mu_{i}\left(\widetilde{M}_{m o}\right)+\lambda_{\min }(\delta(\widetilde{M})) \leqslant \lambda_{i}\left(\widetilde{M}_{m s}\right) \leqslant \mu_{i}\left(\widetilde{M}_{m o}\right)+\lambda_{\max }(\delta(\widetilde{M}))
$$

We have also: $\lambda_{1} \simeq \mu_{1}$ and $\lambda_{n} \simeq \mu_{n}$, so $\lambda_{\min }(\delta(\widetilde{M})) \simeq 0$ and $\lambda_{\max }(\delta(\widetilde{M})) \simeq 0$. From this we can conclude that: For all $i \in\{1,2,3, \ldots, n\}$ :

$$
\lambda_{i} \simeq \mu_{i} \text {. }
$$

and there is $\epsilon, \epsilon$ "small" such that : $\left|\lambda_{i}-\mu_{i}\right| \leqslant \epsilon$ for all $i \in\{1,2,3, \ldots, n\}$.

We now give an important result:

Corollary 1. The market is almost everywhere a "good" approximation of the fundamental value.

For instance the market price of a share is almost everywhere a good approximation of the true value of this share.

Proof. Directly from Theorem above.

\section{Bubbles and crisis}

A single system market bubble is a period market time $B \subseteq I$ such that for all $t \in B s_{i} \neq o\left(o_{i}\right)$ for some $i \in\{1,2,3, \ldots k\}$.

A single system market crisis is a period market time $C \subseteq I$ such that for all $t \in C$ $o_{i}=o\left(s_{i}\right)$ for all $i \in\{1,2,3, \ldots k\}$.

Remark 4. These definitions are not in contradiction with the axiom 5, they cause just that the periods of time $B$ and $C$ are negligible.

Proposition 3. Let $C$ be a period of market time crisis, then $f(\mathbf{p} ; t) \sim f(\mathbf{s} ; t)$.

Proof. The proof is similar than proposition 2.

\section{Example}

Let $m s$ be a market system, and let $\left\{e_{i}, i \in\{1,2, \ldots n\}\right\}$ be a set of expert agents who have an expertise on $m s$. We will suppose that:

- any $e_{i}$ has the same valuation domain

This means that every agent has the same access to the same information available about the market

- all agents are pairwise independent 
The set of expert agents $\left\{e_{i}, i \in\{1,2, \ldots n\}\right\}$ will be represented by a set of vectors with the same name. Since all $e_{i}$ have the same valuation domaine (i. e. the same information about the market) these vectors are unitary; since all agents are pairwise independent these vectors are orthogonal. So these vectors stand as an orthonormal basis $B$. Consequently

$$
m s=\sum_{i} \beta_{i} e_{i}
$$

Their estimation of a quantifiable property $M$ of the market system are eigenvalues. Consequently $B$ is a basis of eigenvectors. Hence let $e_{i}$ be an observer and let $\lambda_{i}$ be its estimation of a quantifiable property $M$ of the market system; Then the probability that the result of the measurement of the quantifiable property $M$ at the time $t$ will be $\lambda_{i}$ is $\sum_{i=1}^{\mu_{\lambda_{i}}}\left|\beta_{i}\right|^{2}$.

\section{Conclusion}

The evolution of financial markets is a challenge for mathematical economics. The multiplication of trades, the very quick evolution of prices, the individual know-how of rational economic agents acting in situation of more or less perfect knowledge, the permanent flow of financial and economic information must be at the heart of economic models. It appears that widespread microeconomic models of financial markets and usual asset pricing models are not fully able to give an accurate representation of financial reality. This is why we propose a mathematical framework which is able to integrate all those dimensions of reality stated above.

The next step will be to simulate numerically the working of a market system such as it is introduced for example in section 5 . We do believe that it may be fitted so as to be close to actual financial markets.

\section{References}

[1] D. Abreu and M. K. Brunnermeier. Bubbles and Crashes, Econometrica, vol. 71, No. 1, 2003.

[2] Bailey R. E., 2007, The Economics of Financial Markets, Cambridge University Press

[3] Fama E., Fisher L., Jensen M., Roll R., 1969 "The Adjustment of Stock Prices to New Information", International Economic Review, Vol. 10, No. 1

[4] Franklin J. N., 1993, Matrix Theory, Dover Publications.

[5] Frydman R., Goldberg M. D., 2010, "The Imperfect Knowledge Imperative in Modern Macroeconomics and Finance Theory", Conference on Microfoundations for Modern Macroeconomics, Columbia University.

[6] Golub G. H., Van Loan C. F, 1996, Matrix computations, Johns Hopkins University Press, third edition.

[7] Kahneman D, Tversky A., 1979, "Prospect Theory: An Analysis of Decision Under Risk", Econometrica, Vol. 47. 
[8] Lang S., 1987, Linear algebra; Undergraduate texts in mathematics, Springer, third edition.

[9] Lang S., 2002, Algebra; graduate texts in mathematics, Springer, third edition.

[10] Lal Mehta M., 2004, Random matrices (3rd edition). Pure and Applied Mathematics Series 142, Elsevier.

[11] Merton R. C., 1998, "Applications of Option-Pricing Theory: Twenty-Five Years Later", The American Economic Review, Vol. 88, No. 3

[12] Muth J. F., 1961, "Rational Expectations and the Theory of Price Movements", Econometrica, Vol. 29

[13] Rudin W., 1987, Real and Complex Analysis, International Series in Pure and Applied Mathematics, third edition Mcgraw Hill book co. New York.

[14] Weihrauch K., 2000, Computable Analysis: An Introduction, Springer-Verlag Berlin/Heidelberg, 